\title{
Intervenções educativas e a dorsalgia numa Unidade de Saúde: um relato de experiência
}

\author{
Educational interventions and back pain in a Health Unit: an experience report \\ Intervenciones educativas y dolor de espalda en una Unidad de Salud: relato de experiencia
}

Recebido: 03/11/2021 | Revisado: 11/11/2021 | Aceito: 16/11/2021 | Publicado: 26/11/2021

\author{
Rosemary de Medeiros Wolter \\ ORCID: https://orcid.org/0000-0001-6210-8701 \\ Universidade Federal de Santa Catarina, Brasil \\ E-mail: dr.rosemarymw@gmail.com \\ Sabrina Blasius Faust \\ ORCID: https://orcid.org/0000-0003-2904-7964 \\ Universidade Federal de Santa Catarina, Brasil \\ E-mail: sabrinabfaust@gmail.com \\ Lenna Eloisa Madureira Pereira \\ ORCID: https://orcid.org/0000-0001-9326-0538 \\ Universidade Federal de Santa Catarina, Brasil \\ E-mail: lenna.madureira@gmail.com
}

\begin{abstract}
Resumo
Relatar a experiência acadêmica em intervenções educativas relativas a lombalgia na unidade de saúde Três Barras. Trata-se de um estudo qualitativo, do tipo relato de experiência, relatando ações desenvolvidas na Unidade de Saúde Três Barras no município de Agrolândia em Santa Catarina, nos meses de julho e agosto de 2020, com pacientes que relataram queixas de lombalgia. A realização das práticas de autocuidado como atividades físicas, alongamentos e a reeducação alimentar formam hábitos que reduziram a prevalência do quadro de dorsalgia. Todos os que fizeram uma reforma no estilo de vida aderindo à uma dieta de alimentos integrais, aumentando o consumo de frutas, verduras, aumentando a ingesta hídrica, bem como integrando atividades físicas leves e alongamentos, obtiveram alívio da lombalgia com redução significante da queixa. Os mesmos reduziram a procura da atenção básica e especializada, solicitaram menos atestado médico e fizeram uso de menos medicamentos. Alguns fatores que agravam as instabilidades da coluna lombar podem ser manejados pelo próprio usuário, trazendo assim alívio parcial ou completo do quadro. A intervenção educativa com enfoque nas ações pró-ativas são de vital importância no manejo desses pacientes, já que podem formar hábitos que reduzem a prevalência da lombalgia, reduzindo assim os atendimentos por essa queixa.
\end{abstract}

Palavras-chave: Educação em saúde; Coluna vertebral; Condições de trabalho; Promoção da saúde; Saúde pública.

\begin{abstract}
To report the academic experience in educational interventions related to low back pain at the Três Barras health unit. This is a qualitative study, of the experience report type, reporting actions developed at the Três Barras Health Unit in the municipality of Agrolândia in Santa Catarina, in the months of July and August 2020, with patients who reported complaints of low back pain. The performance of self-care practices such as physical activities, stretching and dietary re-education form habits that have reduced the prevalence of back pain. All those who made a lifestyle reform adhering to a diet of whole foods, increasing consumption of fruits and vegetables, increasing water intake, as well as integrating light physical activities and stretching, obtained relief from low back pain with a significant reduction in the complaint. They reduced the demand for primary and specialized care, requested less medical certificate and used fewer medications. Some factors that aggravate the instabilities of the lumbar spine can be managed by the user, thus bringing partial or complete relief from the condition. Educational intervention with a focus on proactive actions is of vital importance in the management of these patients, as they can form habits that reduce the prevalence of low back pain, thus reducing care for this complaint.
\end{abstract}

Keywords: Health education; Spine; Working conditions; Health promotion; Public health.

\section{Resumen}

Informar sobre la experiencia académica en intervenciones educativas relacionadas con la lumbalgia en la unidad de salud de Três Barras. Se trata de un estudio cualitativo, tipo relato de experiencia, que reporta acciones desarrolladas en la Unidad de Salud Três Barras en el municipio de Agrolândia en Santa Catarina, en los meses de julio y agosto de 2020, con pacientes que reportaron quejas de lumbalgia. La realización de prácticas de autocuidado como la actividad física, el estiramiento y la reeducación dietética forman hábitos que han reducido la prevalencia del dolor de espalda. 
Todos aquellos que realizaron una reforma de estilo de vida adhiriéndose a una dieta de alimentos integrales, aumentando el consumo de frutas y verduras, aumentando la ingesta de agua, así como integrando actividades físicas ligeras y estiramientos, obtuvieron alivio del dolor lumbar con una reducción significativa de la dolencia. Redujeron la demanda de atención primaria y especializada, solicitaron menos certificado médico y utilizaron menos medicamentos. Algunos factores que agravan las inestabilidades de la columna lumbar pueden ser manejados por el usuario, trayendo así un alivio parcial o total de la condición. La intervención educativa con foco en acciones proactivas es de vital importancia en el manejo de estos pacientes, ya que pueden formar hábitos que reduzcan la prevalencia de lumbalgia, reduciendo así la atención de esta dolencia.

Palabras clave: Educación en salud; Columna vertebral; Condiciones de trabajo; Promoción de la salud; Salud pública.

\section{Introdução}

Lombalgia é usualmente definida como dor localizada abaixo da margem das últimas costelas (margem costal) e acima das linhas glúteas inferiores com ou sem dor nos membros inferiores (Middelkoop et al., 2010). Pode ser aguda, de início súbito e duração menor do que seis semanas, subaguda com duração de seis a doze semanas, e crônica com duração maior do que 12 semanas.

Cerca de $90 \%$ trata-se de lombalgia mecânica comum ou lombalgia inespecífica, quer dizer, ausência de alteração estrutural, e ocorre em todas as faixas etárias. O quadro pode causar limitação das atividades diárias e incapacidade de trabalho temporária, geralmente ocorre desequilíbrio entre a carga funcional, que é o esforço requerido para atividades do trabalho e da vida diária, e a capacidade, que é o potencial de execução para essas atividades (Lizier; Perez \& Sakata, 2012).

A lombalgia é uma das queixas mais comuns no atendimento na Atenção Básica de Saúde e acomete cerca de $70 \%$ a 85\% dos adultos. A prevalência dessa síndrome é de 60-85\% durante a sobrevida dos indivíduos (Haeffner et al., 2015). Dentre os fatores que podem estar relacionados à etiologia desse problema, se encontram: idade, nível educacional, tabagismo, fatores emocionais, obesidade, esforços físicos pesados, movimentos repetitivos e posturas estáticas frequentes (Hoogendoorn et al., 2000).

No público feminino, problemas crônicos de coluna (PCC) acometem cerca de 14,8\% das mulheres de idade fértil e está associada à autopercepção negativa de saúde, bem como o avanço da idade, relacionamento com cônjuge/companheiro, tabagismo, multiparidade, sobrepeso e obesidade e diagnóstico de depressão (Oliveira et al., 2020).

Segundo o Sistema Único de Informações de Benefícios e dos Anuários Estatísticos da Previdência Social, a dor lombar inespecífica é a primeira causa de invalidez entre as aposentadorias previdenciárias e acidentárias. (Filho \& Silva, 2011). A prevalência da dor lombar possui uma alta taxa de prevalência anual $>50 \%$ em indivíduos adultos, entre $13,1 \%$ e 19,5\% nos adolescentes, e valores de prevalência para dor lombar crônica entre 4,2\% e 14,7\% da população brasileira (Nascimento \& Costa, 2015).

O município de Agrolândia onde a experiência foi realizada conta com duas principais fontes de renda, agricultura e confecção, atividades essas que tendem a produzir instabilidades na coluna lombar devido às longas horas de jornada de trabalho, má postura, movimentos repetitivos e sobrecarga. O setor de costura é predominantemente feminino, sexo com predisposição a apresentar problemas crônicos de coluna como já citado acima. Foi constatado durante a atuação na Unidade de Saúde que as queixas entre essas trabalhadoras é muito frequente no município, sendo umas das principais causas de procura do atendimento de saúde.

Visando a redução do afastamento temporário do trabalho, aposentadorias precoces, demanda exagerada do serviço de saúde, uso exagerado de medicamentos analgésicos, anti-inflamatórios e ansiolíticos, que demandam altos custos ao sistema de saúde (Opas, 2016) pode-se empregar técnicas educativas em saúde que restauram a funcionalidade, oferecendo informações relevantes e apoiando a reeducação dos hábitos diários. A Atenção Primária desempenha, neste contexto, seu papel na prevenção das comorbidades comuns à população que busca seus serviços. 
A educação em saúde, por meio de ações teóricas e práticas sobre um determinado assunto de saúde relevante à comunidade local comprova ser um pilar que constrói pessoas autossuficientes e capazes de comprometimento no seu papel referente ao contexto da saúde pessoal e coletivo, sendo fundamental incentivar métodos de promoção de saúde, informando e capacitando as pessoas a tomarem decisões que promovam seu bem estar pessoal, bem como transformando-os em vetores de informações para o seu entorno (Martins et al., 2021).

Desta forma, ela consegue influenciar nas ações destes indivíduos promovendo o empoderamento em relação a um problema comum em seus meios, gera o compartilhamento de conhecimentos e experiências com colegas e pessoas de seu círculo social (Viçosa et al., 2020). O objetivo deste estudo foi relatar a experiência profissional de uma médica, durante sua atuação no Programa Mais Médicos, relativa a atendimentos de pacientes com lombalgia numa unidade básica de saúde.

\section{Metodologia}

Trata-se de estudo qualitativo, do tipo relato de experiência. O relato de experiência é uma ferramenta da pesquisa descritiva que evidencia uma ação que aborda uma situação vivenciada no ambiente profissional de interesse da comunidade científica (Flick, 2013). O relato da experiência está além de uma descrição sucinta sobre algum fato ou evento, pois ao realizar a sua leitura, é verossímil compreender com mais fidedignidade a experiência abordada (Martins et al., 2021).

Esta experiência aconteceu na unidade básica de saúde de Três Barras (SC), durante o período de atuação da profissional médica no Programa Mais Médicos (PMM) e realização do primeiro ciclo de atividades do Programa que conta com a especialização em Atenção Básica. Esta atividade foi realizada no primeiro semestre de 2020, e teve a parceria de toda equipe multiprofissional da unidade de saúde de Três Barras, composta por enfermeira, técnica e Agentes Comunitários de Saúde (ACS), além da cooperação da nutricionista e fisioterapeuta. Foi realizado um mapeamento das necessidades dos usuários frequentadores da unidade de saúde, pela médica, e foi diagnosticado principalmente a questão da lombalgia em profissionais que atuam na área da costura.

A intervenção educativa pensada foi a elaboração do banner, um folheto e um plano de acompanhamento em forma de questionário aos usuários da unidade de saúde que relatam queixas de lombalgia. O banner se constitui de informações sobre a definição de lombalgia, fatores de risco e instruções concisas sobre prevenção e manejo. Inclui também imagens explicativas sobre os mesmos temas para melhor assimilação, como mostra a Figura 1. O folheto sobre dorsalgia contém informações sobre atividades físicas, alongamento e reeducação alimentar acompanhando imagens ilustrativas.

Figura 1. Folheto sobre dorsalgia.

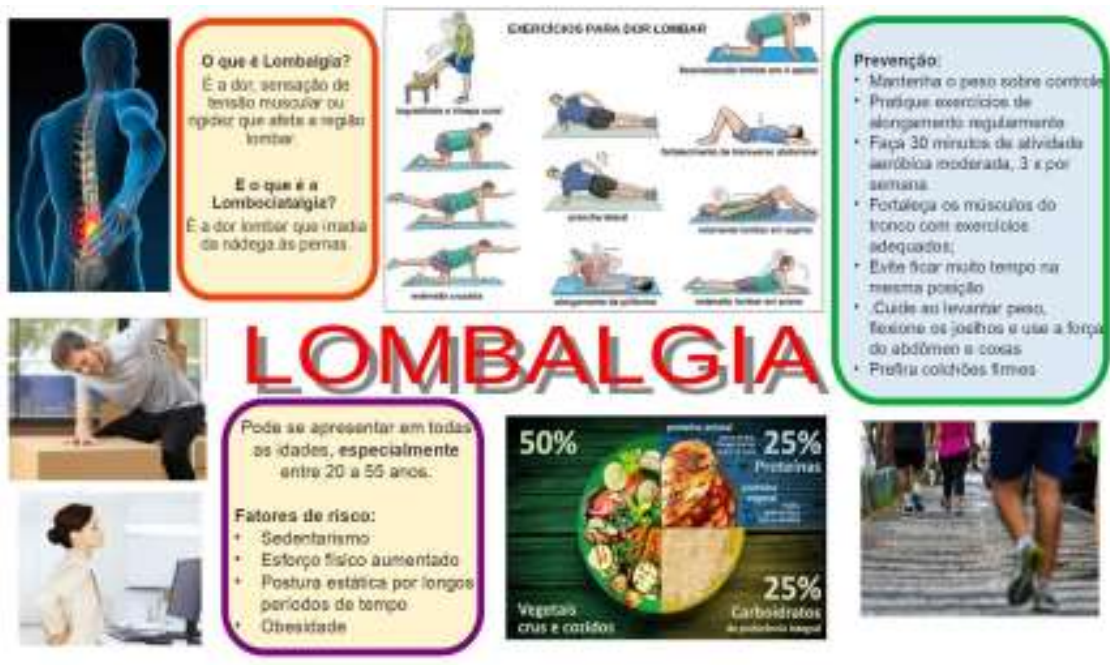

Fonte: Autores (2020). 
Devido a impossibilidade de palestras e organização de grupos presenciais no período de pandemia, os pacientes sintomáticos foram abordados na triagem com os profissionais da unidade, e durante a consulta médica com a relatora, na finalidade de motivá-los a realizarem um programa de reeducação alimentar, alongamento e atividade física.

\section{Resultados e Discussão}

A proposta posta em prática foi no sentido de apresentar intervenções educativas relativas à dorsalgia de coluna por meio de materiais educativos e orientadores sobre prevenção e manejo do problema já instalado. A expectativa foi de que por meios educativos e de apoio à mudança do estilo de vida, os usuários da unidade conseguissem adotar uma postura proativa em relação ao problema, reduzindo possíveis causas para a dorsalgia como: postura de trabalho, sedentarismo, obesidade.

Terapias como a auriculoterapia podem ser utilizadas para alívio e sintomas da lombalgia. No estudo experimental numa cadeia pública do Mato Grosso a prática foi realizada nos profissionais do sistema penitenciário, mostrando como a auriculoterapia é promissora aos benefícios e redução das dores, na disposição do exercício laboral e uma melhor qualidade de vida (Graça et al., 2020).

A realidade dos trabalhadores da América do Sul mostra o quanto a saúde laboral precisa de esclarecimentos e sensibilização. No caso do Peru, trabalhadores relataram que suas posturas são incômodas ou forçadas, com movimentos repetitivos, devido às características do trabalho com jornadas laborais extensas e com enfermidades causadas por este tipo de trabalho (Sabastizagal-Vela, Astete-Cornejo \& Benavides, 2020).

No exemplo da Espanha (Ramirez Huaranga, De La Rocha Vedia, Plasencia Ezaine et al. 2019), o valor da sintomatologia no tratamento da Lombalgia crônica foi constatado que fatores psicológicos são determinantes para obtenção de melhores resultados terapêuticos. Dados de estudo canadense apontou que tratamentos de nível multidisciplinar, abrangendo aspectos psicológicos, farmacológicos e de fisioterapia contribuíram para a diminuição da dor. A positividade foi item detectado no estudo suíço para intervenções da coluna na redução da dor.

Um dos fatores associados à lombalgia reside no fator obesidade. Tal confirmação pode ser vista no estudo de EspíLópez et al. (2019) onde com base na busca sistematização de dados puderam confirmar a relação direta da obesidade e sobrepeso com a manifestação e aumento da lombalgia. A promoção de hábitos e estilo de vida saudável são apontados como fatores de prevenção da lombalgia.

O controle da obesidade com manejo multidisciplinar traz melhor resultado para pessoas com dor lombar, é o que comprova o estudo observacional de Bravo Peña et al. (2020) ao constatar a eficiência ou fracasso no tratamento da dor lombar axial. Os resultados foram promissores para pessoas que conseguiram melhor controlar a obesidade.

Analisando-se os estudos acima é possível afirmar que independente da terapêutica escolhida, a informação e educação é essencial para que o paciente contribua com todo o processo de manejo e/ou cura do quadro. Uma das estratégias que promovem a responsabilidade social é a Educação em Saúde, que é compreendida como a associação de atitudes e experiências de aprendizado com intuito de promover tranquilizar o paciente, esclarecer dúvidas e orientar sobre a participação ativa que lhe é devida no cuidado de sua saúde laboral (Martins et al., 2021).

A realização das práticas de autocuidado como atividades físicas, alongamentos e a reeducação alimentar formam hábitos que reduzem a prevalência do quadro de dorsalgia, contribuindo assim para a redução do uso repetitivo dos serviços de saúde, emissão de atestados médicos frequentes, uso indiscriminado de analgésicos, anti-inflamatórios e ansiolíticos.

\section{Conclusão}

Na Unidade Básica de Saúde Três Barras foi realizada intervenção educativa relativa à perda de peso, atividade física e alongamento para o alívio e redução das queixas de lombalgia. Por meio de um instrumento - um banner educativo e folheto 
explicativo - o primeiro usado em consultório para orientação e o último entregue aos pacientes com dor crônica de coluna como principal queixa. Foi abordada a necessidade de aumentar o consumo de frutas e verduras, ingestão hídrica e iniciação da atividade física leve acessível a todos, a exemplo da caminhada. Foi orientado também alongamentos que podem ser realizados diariamente no domicílio e nos intervalos nos ambientes de trabalho.

É possível perceber que para abordarmos o quadro de lombalgia e dores de coluna em geral faz-se necessário pensarmos em nível multidisciplinar, como: educação de postura no ambiente de trabalho ou ao realizar atividades diárias, melhora do estilo de vida visando alimentação saudável, a perda de peso visto que a obesidade é um fator preponderante na piora do quadro; e o estado emocional que por sua vez, também mantém influência no quadro de lombalgia. Algumas terapias não convencionais, como a Auriculoterapia podem contribuir para o alívio e bem estar das pessoas que convivem com a dorsalgia.

A educação em saúde é importante nesta temática, pois consegue remover o foco exclusivo aos tratamentos medicamentosos e cuidado especializado, sendo apresentado a responsabilidade do autocuidado ao paciente. A educação em saúde é capaz de empoderar o usuário de conhecimentos para alívio e redução das crises de dor e incapacidade física. Os esforços e recursos direcionados pela educação podem se traduzir na diminuição da busca em casos agudos ao sistema de saúde, um controle ao tratamento crônico, além do bem estar geral destes pacientes.

Na experiência na Unidade Básica de Saúde Três Barras os pacientes que colocaram em prática as orientações apresentadas, relataram uma melhora significativa e passaram a procurar o atendimento médico com menos frequência. Apesar da atividade ter sido realizada com um número reduzido de pessoas em virtude do período de Pandemia de Covid-19, concluiuse que os fatores abordados via educação em saúde conseguiram reformar o estilo de vida destes usuários.

Entretanto, há necessidade de trabalhos e estudos futuros sobre a avaliação de continuação das técnicas educativas na unidade de saúde, seja por meio de informações verbais e/ou outros exemplos práticos validando os conceitos de promoção da saúde dos adultos por meio de suas escolhas e relatos pessoais. No cotidiano do atendimento é comum pessoas solicitarem uma conduta similar ao do familiar ou amigo que obteve melhoras com o tratamento.

Quanto ao uso de medicamentos e a diminuição da emissão de atestados médicos e consequente encaminhamentos aos serviços de especialidades, foi observada uma relativa diminuição pela equipe de assistência à saúde na atenção primária, não chegando a ser comprovado por dados estatísticos o que pode ser entendido como a limitação deste relato, necessitando assim de estudos mais detalhados para avaliar a real diminuição dos casos.

\section{Referências}

Bravo Peña, M. E., Bravo Peña, M. E., Arias Botero, J. H., Buritica Franco, J., Salazar Galeano, C., \& Yepes Rios, A. F. (2020). Fallo terapéutico del bloqueo de ramo medial en el dolor facetario lumbar y su relación con la obesidad. Estudio de cohorte retrospectivo. Revista de La Sociedad Española Del Dolor, 27. https://doi.org/10.20986/resed.2020.3829/2020

Cormier, S., Lavigne, G. L., Choinière, M., \& Rainville, P. (2016). Expectations predict chronic pain treatment outcomes. PAIN, 157(2), 329-338. https://doi.org/10.1097/j.pain.0000000000000379

Espí-López, G. V., Muñoz-Gómez, E., Arnal-Gómez, A., Fernández-Bosch, J., Balbastre-Tejedor, I., Ramírez-Iñiguez, M. V., Vicente-Herrero, M. T., EspíLópez, G. V., Muñoz-Gómez, E., Arnal-Gómez, A., Fernández-Bosch, J., Balbastre-Tejedor, I., Ramírez-Iñiguez, M. V., \& Vicente-Herrero, M. T. (2019). La obesidad como factor determinante en el dolor lumbar: revisión bibliográfica. Revista de La Asociación Española de Especialistas En Medicina Del Trabajo, 28(3), 217-228. https://scielo.isciii.es/scielo.php?script=sci_arttext\&pid=S1132-62552019000300006

Filho, N. M., \& Silva, G. A. e. (2011). Invalidez por dor nas costas entre segurados da Previdência Social do Brasil. Revista de Saúde Pública, 45(3), 494-502. https://doi.org/10.1590/s0034-89102011000300007

Flick, U. (2010). Introducing Research Methodology: A Beginner's Guide to Doing a Research Project. Los Angeles: Sage. MANUSYA, 13(1), 81-82. https://doi.org/10.1163/26659077-01301006

Graça, B. C. da, Nascimento, V. F. do, Felipe, R. N. R., Andrade, A. C. de S., Atanaka, M., \& Terças-Trettel, A. C. P. (2020). Use of auriculotherapy to control low back pain, anxiety and stress of professionals of the correctional system. Brazilian Journal of Pain. https://doi.org/10.5935/2595-0118.20200025

Haeffner, R., Sarquis, L. M. M., Haas, G. F. da S., Heck, R. M., \& Jardim, V. M. da R. (2015). Prevalência de lombalgia e fatores associados em trabalhadores 
Research, Society and Development, v. 10, n. 15, e256101522758, 2021

(CC BY 4.0) | ISSN 2525-3409 | DOI: http://dx.doi.org/10.33448/rsd-v10i154.22758

de uma empresa agropecuária do sul do Brasil. Revista Brasileira de Medicina Do Trabalho, 13(1), 35-42. http://www.rbmt.org.br/details/25/pt$\mathrm{BR} /$ prevalencia-de-lombalgia-e-fatores-associados-em-trabalhadores-de-uma-empresa-agropecuaria-do-sul-do-brasil

Hoogendoorn, W. E., van Poppel, M. N. M., Bongers, P. M., Koes, B. W., \& Bouter, L. M. (2000). Systematic Review of Psychosocial Factors at Work and Private Life as Risk Factors for Back Pain. Spine, 25(16), 2114-2125. https://doi.org/10.1097/00007632-200008150-00017

Lizier, D. T., Perez, M. V., \& Sakata, R. K. (2012). Exercícios para tratamento de lombalgia inespecífica. Revista Brasileira de Anestesiologia, 62(6), 842846. https://doi.org/10.1590/s0034-70942012000600008

Martins, L. G. de L., Pimentel, I. M. de S., Panzetti, T. M. N., Ferreira, I. P., Negrão, R. de J. da S., Boulhosa, M. F., Carvalho, D. S. de, Dias, N. M., Silva, L. da S. e, Silva, C. de M. da, Coutinho, A. C. de O., Ferreira, F. dos S., Brito, A. R., Pinto, J. V. C., \& Cardoso, M. Q. (2021). Educação em saúde sobre a dengue em uma escola: Relato de experiência. Research, Society and Development, 10(7), e36510716291. https://doi.org/10.33448/rsd-v10i7.16291

Middelkoop, M. V., Rubinstein, S. M., Verhagen, A. P., Ostelo, R. W., Koes, B. W., \& van Tulder, M. W. (2010). Exercise therapy for chroni/c nonspecific low-back pain. Best Practice \& Research Clinical Rheumatology, 24(2), 193-204. https://doi.org/10.1016/j.berh.2010.01.002

Nascimento, P. R. C. do, \& Costa, L. O. P. (2015). Prevalência da dor lombar no Brasil: uma revisão sistemática. Cadernos de Saúde Pública, 31(6), 11411156. https://doi.org/10.1590/0102-311x00046114

Oliveira, C. V. de A. et al. (2020). Prevalência e fatores associados a problema crônico de coluna em mulheres em idade fértil. Ciência \& Saúde Coletiva [online], 25 (3), 1041-1049. https://doi.org/10.1590/1413-81232020253.15522018

Organização Panamericana da Saúde. (2015). Uso racional de medicamentos: fundamentação em condutas terapêuticas e nos macroprocessos de Assistência Farmacêutica. Uso Racional de Medicamentos: Fundamentação Em Condutas Terapêuticas E Nos Macroprocessos de Assistência Farmacêutica, 179-179. https://pesquisa.bvsalud.org/bvsms/resource/pt/mis-40335

Ramirez Huaranga, M. A., De la Rocha Vedia, I. V., Plasencia Ezaine, A. Es., Jaramillo Tascon, C. A., Calle Ochoa, J. H., \& LÓPEZ LÓPEZ, A. (2019). El papel de la sintomatología depresiva, catastrofismo y expectativa en la eficacia de las técnicas intervencionistas para el tratamiento del dolor lumbar crónico. Revista de La Sociedad Española Del Dolor. https://doi.org/10.20986/resed.2019.3704/2018

Sabastizagal-Vela, I., Astete-Cornejo, J., \& Benavides, F. G. (2020). Condiciones de trabajo, seguridad y salud en la población económicamente activa y ocupada en áreas urbanas del Perú. Revista Peruana de Medicina Experimental Y Salud Pública, 37(1), 32-41. https://doi.org/10.17843/rpmesp.2020.371.4592

Viçosa, D. L., Viçosa, C. S. C. L., Santana, E. B., Carvalho, M. T. X., Folmer, V., \& Salgueiro, A. C. F. (2020). Postural education as strategy for health promotion at school. Research, Society and Development, 9(2), e29921965. https://doi.org/10.33448/rsd-v9i2.1965 